\title{
The effect of continuous versus intermittent renal replacement therapy on the outcome of critically ill patients with acute renal failure (CONVINT): a prospective randomized controlled trial
}

\author{
Joerg C Schefold ${ }^{1 *}$, Stephan von Haehling ${ }^{2}$, Rene Pschowski ${ }^{1,3}$, Thorsten Onno Bender ${ }^{1}$, Cathrin Berkmann ,
} Sophie Briegel ${ }^{1}$, Dietrich Hasper ${ }^{1}$ and Achim Jörres $^{1}$

\begin{abstract}
Introduction: Acute renal failure (ARF) requiring renal replacement therapy (RRT) occurs frequently in ICU patients and significantly affects mortality rates. Previously, few large clinical trials investigated the impact of RRT modalities on patient outcomes. Here we investigated the effect of two major RRT strategies (intermittent hemodialysis (IHD) and continuous veno-venous hemofiltration $(\mathrm{CWH})$ ) on mortality and renal-related outcome measures.

Methods: This single-center prospective randomized controlled trial ("CONVINT") included 252 critically ill patients (159 male; mean age, $61.5 \pm 13.9$ years; Acute Physiology and Chronic Health Evaluation (APACHE) II score, $28.6 \pm 8.8$ ) with dialysis-dependent ARF treated in the ICUs of a tertiary care academic center. Patients were randomized to receive either daily IHD or CWH. The primary outcome measure was survival at 14 days after the end of RRT. Secondary outcome measures included 30-day-, intensive care unit-, and intrahospital mortality, as well as course of disease severity/biomarkers and need for organ-support therapy.

Results: At baseline, no differences in disease severity, distributions of age and gender, or suspected reasons for acute renal failure were observed. Survival rates at 14 days after RRT were 39.5\% (IHD) versus 43.9\% (CVH) (odds ratio (OR), $0.84 ; 95 \%$ confidence interval $(C I), 0.49$ to $1.41 ; P=0.50$ ). 14-day-, 30-day, and all-cause intrahospital mortality rates were not different between the two groups (all $P>0.5$ ). No differences were observed in days on RRT, vasopressor days, days on ventilator, or ICU-/intrahospital length of stay.

Conclusions: In a monocentric RCT, we observed no statistically significant differences between the investigated treatment modalities regarding mortality, renal-related outcome measures, or survival at 14 days after RRT. Our findings add to mounting data demonstrating that intermittent and continuous RRTs may be considered equivalent approaches for critically ill patients with dialysis-dependent acute renal failure.
\end{abstract}

Trial registration: NCT01228123, clinicaltrials.gov

\footnotetext{
* Correspondence: schefold@charite.de

'Department of Nephrology and Medical Intensive Care,

Charité- Universitätsmedizin Berlin, Campus Virchow-Klinikum, Berlin,

Germany

Full list of author information is available at the end of the article
} 


\section{Introduction}

Acute renal failure (ARF) requiring intermittent or continuous renal replacement therapy (RRT) significantly affects morbidity and mortality of critically ill patients and constitutes a substantial health care burden [1]. This remains to be the case despite manifold improvements in RRT techniques and after substantial advances in supportive ICU care. Importantly, development of ARF constitutes an independent risk factor for death in the ICU, and data indicate that early induction of RRT significantly improves the prognosis of affected patients [1-4].

Continuous RRT (CRRT) is widely used in ICUs and is often viewed as the preferable approach in critically ill ARF patients. It remains, however, unclear whether the choice of initial RRT modality may affect patient outcomes, as only few prospective randomized controlled trials (RCTs) have directly compared the different approaches [5-10]. Often these studies were, however, either small and/or had methodologic or randomization problems. In addition, heterogeneous patient cohorts were included (surgical/medical) and different CRRT modalities (convective, diffusive, or both) applied. Only two larger multicenter RCTs directly compared IHD and CVVHD [10] or IHD and CVVH [7]. On the whole, meta-analysis of available data did not demonstrate a general survival benefit for either strategy [3,11-15]. Even fewer data are available regarding end points such as time to hospital/ICU death or discharge, survival at 14 days after RRT, or specific renal-related outcome measures.

To identify an RRT option of choice would potentially have a major impact on clinical procedures. This might be of particular importance in sepsis-induced renal injury [16] and might also affect treatment costs [17]. We therefore embarked on a single-center prospective randomized controlled trial ("CONVINT") that set out to elucidate further the potential impact of initial choice of RRT modality by studying a cohort of 252 critically ill patients with ARF in medical ICU of a tertiary care academic center.

\section{Materials and methods}

\section{Design, study patients, and inclusion/exclusion criteria}

Patients with ARF requiring RRT treated at the medical intensive care units (ICUs) of the Charite University Hospital, Campus Virchow-Klinikum, Department of Nephrology and Medical Intensive Care, Berlin, Germany, were included in this randomized controlled trial (CONtinuous Vs. INTermittent RRT on the outcome of critically ill patients with ARF trial; CONVINT). CONVINT was performed from January 2002 until October 2007. A single-center prospective randomized controlled openlabel trial design applied.

During the recruitment period, adult ( $>18$ years) patients with ARF requiring RRT were eligible for inclusion in CONVINT. Need for RRT was defined as presence of at least one of the following criteria: (a) clinical symptoms of uremia (that is, gastrointestinal symptoms: (for example, nausea, vomiting, diarrhea not explained otherwise; or neurologic symptoms: mental confusion, severe weakness, seizures/coma not explained otherwise; or evidence of pericardial effusion); (b) persisting oliguria (urinary excretion rate $<0.5 \mathrm{ml} / \mathrm{kg} / \mathrm{min}$ for $>12$ hours), or anuria (anuria for $>12$ hours or $<0.3 \mathrm{ml} / \mathrm{kg} / \mathrm{h}$ for $>24$ hours), despite adequate fluid management; (c) fluid overload not responding to diuretic treatment; (d) blood urea nitrogen (BUN) levels $>100 \mathrm{mg} / \mathrm{dl}$ or creatinine clearance $<0.1 \mathrm{ml} / \mathrm{kg}$ of body weight/min; (e) severe metabolic acidosis $(\mathrm{pH}<7.2)$ not responding to conventional treatment; and (f) hyperkalemia not responding to conservative treatment.

Patients were excluded when any of the following criteria were met: (a) preexisting chronic renal failure with serum creatinine $>3 \mathrm{mg} / \mathrm{dl}$ or patients receiving chronic dialysis; (b) kidney-transplant recipients; (c) patients not requiring ICU treatment; (d) denial of written informed consent (patient, legal representative, or legal proxy). The study was approved by the local Ethics Committee (Ethikkommission der Charité-Universitätsmedizin Berlin) and was designed in adherence to the Declaration of Helsinki. Informed consent to participate in the trial was obtained from the patient or legal representative. In case a patient was unable to give informed consent and no legal representative was available, the Ethics Committee approval permitted immediate inclusion of the patient in the trial and to obtain informed consent subsequently, that is, as soon as a legal representative (next of kin without formal rights of representation not sufficient) was installed or the patient regained ability to give informed consent. Data of patients who died before written informed consent was obtained remained in the evaluation. Before randomization, next of kin were always asked if they agreed to including the patient in the study and using the data for future publication. If they declined, or only expressed concerns regarding potential objections the patient himself might have against study participation/publication of data, this patient was not included in the first place.

\section{Randomization procedure and treatment protocol}

After assessment of inclusion and exclusion criteria, patients were allocated to the respective study groups (group 1, IHD; group 2, CVVH) at study day 0 (day of randomization; 1 ). To assure concealment of allocation, an independent external telephone (computer-based) randomization procedure provided by the Department of Biometry and Medical Documentation, University of Ulm, Ulm, Germany, was used (permutated blocks of four).

IHD (AK100/AK200; Gambro, Lund, Sweden) was performed by using polysulfone synthetic membranes. 
Standard treatment dose was daily IHD with 4 hours of hemodialysis at a blood flow of 200 to $250 \mathrm{ml} / \mathrm{min}$. A dialysate flow at $500 \mathrm{ml} / \mathrm{min}$, volumetric UF-control, water purified by reverse osmosis, and bicarbonate dialysate was used. Postdilutional CVVH (BM11/BM14; Baxter Medical, Deerfield, IL, USA, or Multifiltrate, Fresenius Medical Care, Bad Homburg, Germany) was applied 24 hours daily by using a polysulfone synthetic membrane, blood flow of $200 \mathrm{ml} / \mathrm{min}$, prescribed filtration rate of $35 \mathrm{ml} / \mathrm{kg}$ of body weight/hour, by using bicarbonate-buffered substitution fluids. General treatment target in both groups was absence of any criteria for acute RRT and a target-time-averaged serum urea of 100 to $150 \mathrm{mg} / \mathrm{dl}$ every 72 hours (mean pre + post + pre in IHD; not intended as an outcome measure). $\mathrm{CVVH}$ membranes were exchanged every 24 hours in patients with severe sepsis/septic shock. In all other patients, membranes were exchanged every 48 hours. As defined in the study protocol, patients in both study groups were allowed to be switched to the respective other RRT modality in cases of significant medical reasons (that is, either in cases of severe RRT-modality-associated complications or whenever the other modality should not be withheld for significant medical reasons). In such cases, switching of the respective RRT modality was performed by the attending ICU physician. Typical reasons for switching of RRT modality were as follows IHD to CVVH: continuous severe hypotension with requirement of advanced inotropic support or impossibility of maintaining fluid homeostasis. CVVH to IHD: patient mobilization, impossibility of maintaining electrolyte/ acid base homeostasis, impossibility of delivering an adequate dialysis dose, impossibility of performing CVVH without anticoagulation in patients with bleeding complications/repeated filter clotting. Number of changes in RRT modality and respective reasons were recorded.

\section{Clinical and laboratory follow-up of study patients, outcome measures}

Baseline demographics (including hospital/ICU admission day), baseline laboratory data (including baseline blood-gas analyses, urinary output), concomitant diagnoses, and reasons for ARF were recorded. For assessment of clinical disease severity over time, the following clinical scoring systems were used: Sepsis-related Organ Failure Assessment (SOFA) [18], and Therapeutic Intervention Scoring System (TISS)-28 [19]. Acute Physiology And Chronic Health Evaluation (APACHE) II score [20] and Simplified Acute Physiology Score (SAPS)-2 [21] were recorded at baseline. Data on respective RRT treatments, changes in RRT modality, necessity for mechanical ventilation or vasopressor support, routine laboratory data (daily until study day 10 and at days 15, 21), and outcome-related data were recorded daily. Patients were followed up until 14 days after RRT, withdrawal of consent, or death. Follow-up was performed by using electronic hospital data-management systems or telephone follow-up.

The primary outcome measure of CONVINT was survival at 14 days after end of RRT. Secondary outcome measures included 14-day-, 30-day-, all cause intrahospital mortality, days until death (on ICU), days in ICU/ hospital, days on RRT/dialysis-free days, total days on vasopressors/mechanical ventilation, and course of disease severity (that is, clinical scores).

\section{Statistical analyses}

The initial power calculation aimed at inclusion of $n=200$ patients per arm (the OR of failure (that is, primary end point) between the study groups can be given as the probability (true $\log \mathrm{OR}$ is between estimated $\log \mathrm{OR} \pm 0.45$ ) is at least 0.95 , under the assumption that failure rates of the therapies may vary within $40 \%$ to $80 \%$. No hypothesis of superiority of one of the methods studied, nor equality between the methods, was initially anticipated. Thus, estimation of effects was the focal point. CONVINT was terminated after inclusion of $n=252$ patients in October 2007 after substantial treatment-protocol changes due to replacement of respective RRT equipment provided by the university hospital (change in contract; machines provided by different supplier; please also refer to Discussion section). Data from two of the 252 included patients were lost to follow-up and could not be included in the final analysis. One patient/proxy per study group withdrew consent (data until withdrawal entered the analysis). With a sample size of 125 patients per group, a precision of log $\mathrm{OR} \pm 0.50$ is achieved.

As defined in the study protocol, the sample for the statistical analysis consists of the intention-to treat (ITT) population. Patients were evaluated in the group to which they had been randomly allocated. An explorative PP analysis was performed. Subgroup analyses of patients with need for advanced inotropic support (norepinephrine dose of $>0.3 \mu \mathrm{g} / \mathrm{kg} / \mathrm{min}$ at any time during the study interval; overall sample group) was performed to identify potential outcome differences between the study groups. Switches from one RRT modality to the other were evaluated as a secondary end point. Statistical methods included Student paired and unpaired $t$ test and $\chi^{2}$ test, as appropriate. Kaplan-Meier survival estimates were constructed for illustrative purposes. Data were checked for normal distribution by using the KolmogorovSmirnov test. Results are reported as means \pm standard deviations (SD), if not indicated otherwise. Significance was assigned when $P<0.05$. Statistical analyses were performed by using MedCalc 12.0.1 software (MedCalc Software, Mariakerke, Belgium). 


\section{Results}

\section{Baseline demographics and study groups}

Data from $n=128$ patients (group 1, IHD) and $n=122$ (group 2, CVVH) patients were analyzed (Figure 1). At baseline, no statistically significant differences between the study groups were noted regarding demographics, days on ICU until study inclusion, disease severity (as assessed by APACHE II, SOFA, SAPS-2, and TISS-28 scores), key physiological and hemodynamic indices (except diastolic blood pressure), renal function, and acid/ base balance (Table 1). Concomitant illness was recorded in both study groups. The number of patients with advanced vascular disease, congestive heart failure, chronic obstructive pulmonary disease, and metabolic disease (including diabetes) was not different between the two study groups (Table 1). Major reasons for development of ARF were severe sepsis/septic shock (both groups $>65 \%$ of study patients at baseline) or cardiogenic shock (please also refer to Table 1). At baseline, $>80 \%$ of study patients in both groups required both mechanical ventilation and vasopressor support (Table 1) and thus suffered from multiple organ failure. Main underlying cause for developing severe sepsis/septic shock was pneumonia $(n=33$ patients in group 1 versus $n=25$ patients in group 2, n.s.). Moreover, 22 patients were initially diagnosed with Acute Respiratory Distress Syndrome (ARDS) (group 1, $n=10$; group 2, $n=12 ; P=$ n.s.). Peritonitis was present in $n=5$ (group 1) versus $n=5$ (group 2) patients ( $P=$ n.s.). In addition, $n=9$ (group 1 ) versus $n=8$ patients (group 2) were diagnosed with severe pancreatitis. At baseline, no difference in vasopressor need or need for mechanical ventilation was noted (Table 1).

Follow-up of ARF, need for dialysis, and renal-related outcome measures

The time course of clinical and laboratory data is shown in Table 2. Time until dialysis, days on RRT, dialysis-free days (all n.s.; Tables 1 and 3), as well as course of urinary output, serum creatinine, and serum urea concentrations were not different between the two groups. No differences were noted in serum creatinine at hospital discharge or total fluid balance (Table 3). In the IHD group, the mean blood-flow rate was $222.9 \pm 30.4 \mathrm{ml} / \mathrm{min}$, and mean dialysate flow rate was $491.4 \pm 6.23 \mathrm{ml} / \mathrm{min}$. Mean duration of IHD was $215.4 \pm 82.3 \mathrm{~min} / \mathrm{session}$. In the CVVH group, mean applied dose (that is, filtration rate was $30.9 \pm 7.0 \mathrm{ml} / \mathrm{kg}$ body weight/hour ( $88 \%$ of prescribed dose). Mean blood flow rate in the CVVH group was $188.7 \pm 29.4 \mathrm{ml} / \mathrm{min}$. Mean daily ultrafiltration rates were $1.24 \pm 0.9 \mathrm{~kg}$ (IHD group) and $1.25 \pm 1.2 \mathrm{~kg}(\mathrm{CVVH}$ group) $(P=0.87)$, respectively (means $\pm \mathrm{SD}$ are given). Anticoagulation was used in $72.8 \%$ (IHD) and $74.4 \%$ $(\mathrm{CVVH})$ of sessions (unfractionated heparin used in $99.4 \%$ versus $97.8 \%$ of sessions, respectively). Hirudine derivates or citrate/calcium were used in the remaining cases. Mean dose of unfractionated heparin was $682.8 \pm 357$ i.U./hour (IHD group) versus $781.6 \pm 497$ i.U./hour (CVVH group) $(P=0.0001)$.

As defined in the study protocol, patients in both groups were allowed to cross over to the respective

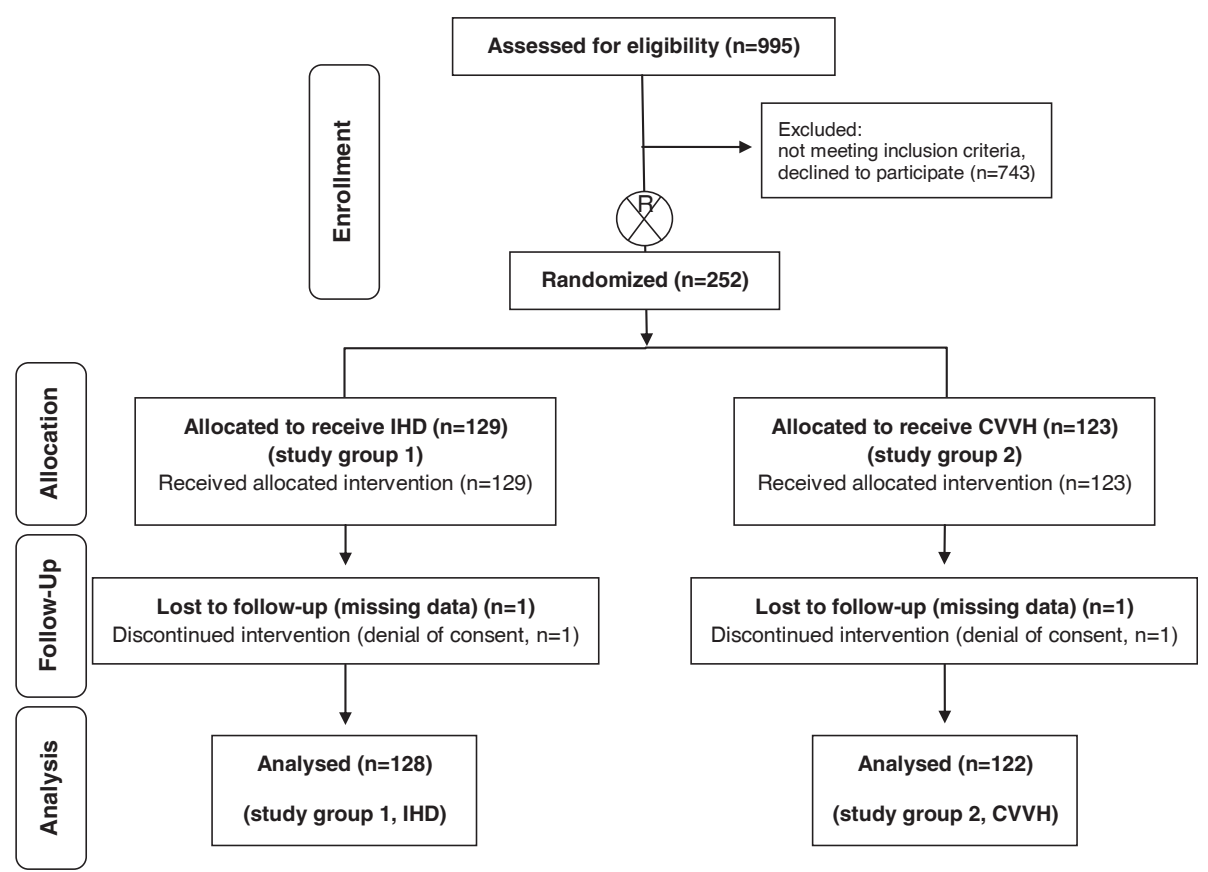

Figure 1 Study flow chart. 
Table 1 Baseline demographics/disease severity, and concomitant diseases

Age (years)
Gender (male)
Major category (nr. of patients)
Body weight (kg)
Temperature ('Celsius)
Hemoglobin (mg/dl)/(hematocrit) (\%)
Serum creatinine (mg/dl)
Serum urea (mg/dl)
Serum potassium (mM)
Baseline blood pH
Baseline HCO 3
Urine output (within 24 hours before randomization; ml)
Days in ICU until randomization
Days from ICU admission until start of RRT
Need for mechanical ventilation (at study day 1 )
paO ${ }_{2} /$ FiO ${ }_{2}$ ratio (in all patients at study day 1)
Need for (any) vasopressor (at study day 1 )
Key hemodynamic variables (at study day 1)

Reason for ARF/need for RRT (number of patients)

APACHE-II score

SAPS-II score

SOFA score

TISS-28 score

Concomitant disease(s) (number of patients; multiple possible)
Medical
Postsurgery
Posttrauma

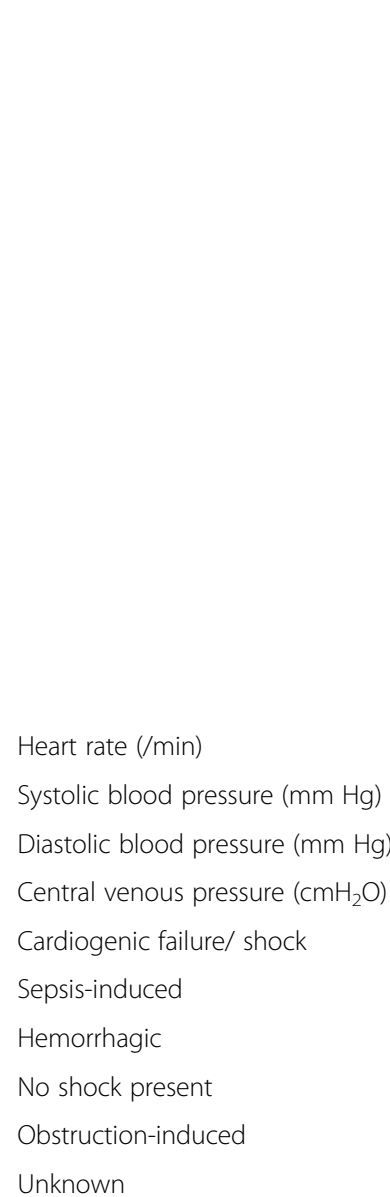

\begin{tabular}{|c|c|c|c|}
\hline & $\begin{array}{l}\text { iHD group } \\
(n=128)\end{array}$ & $\begin{array}{l}\text { CVVH group } \\
\quad(n=122)\end{array}$ & $\begin{array}{c}P \\
\text { value }\end{array}$ \\
\hline & $60.8 \pm 13.4$ & $62.3 \pm 14.5$ & 0.41 \\
\hline & 81 (63.3\%) & 75 (61.5\%) & $0.97^{\mathrm{a} *}$ \\
\hline & $120(93.8 \%)$ & 115 (94.3\%) & $0.95^{\mathrm{a} *}$ \\
\hline & $8(6.3 \%)$ & $5(4.1 \%)$ & $0.66^{\mathrm{a}}$ \\
\hline & 0 & $2(1.6 \%)$ & $0.46^{\mathrm{a}}$ \\
\hline & $82.1 \pm 22.8$ & $86.1 \pm 25.2$ & 0.28 \\
\hline & $37.5 \pm 1.2$ & $37.5 \pm 1.3$ & 0.74 \\
\hline & $10.6 \pm 1.7$ & $10.4 \pm 1.7$ & 0.30 \\
\hline & {$[31.7 \pm 5.8]$} & {$[31.4 \pm 5.4]$} & {$[0.72]$} \\
\hline & $3.64 \pm 2.3$ & $3.57 \pm 1.9$ & 0.81 \\
\hline & $159.7 \pm 86.5$ & $156.7 \pm 77.1$ & 0.77 \\
\hline & $4.66 \pm 0.8$ & $4.65 \pm 0.9$ & 0.91 \\
\hline & $7.32 \pm 0.2$ & $7.32 \pm 0.1$ & 0.82 \\
\hline & $22.6 \pm 6.8$ & $21.8 \pm 5.4$ & 0.34 \\
\hline & $927.1 \pm 1318.4$ & $708.5 \pm 937.8$ & 0.10 \\
\hline & $1.0[0-3.0]^{\mathrm{b}}$ & $1.0[0-2.3]^{b}$ & 0.82 \\
\hline & $1.0[0-4.0]^{\mathrm{b}}$ & $1.0[0-3.0]^{\mathrm{b}}$ & 0.55 \\
\hline & $113(88.3 \%)$ & $103(84.4 \%)$ & 0.88 \\
\hline & $197.3 \pm 107.9$ & $208.7 \pm 106.8$ & 0.45 \\
\hline & $104(81.2 \%)$ & 106 (86.9\%) & $0.79^{a *}$ \\
\hline & $104.0 \pm 26.1$ & $104.7 \pm 20.9$ & 0.81 \\
\hline n Hg) & $111.6 \pm 22.5$ & $109.8 \pm 19.4$ & 0.51 \\
\hline m Hg) & $56.4 \pm 11.1$ & $53.0 \pm 13.6$ & 0.04 \\
\hline $\left.\mathrm{nH}_{2} \mathrm{O}\right)$ & $14.7 \pm 5.3$ & $14.2 \pm 5.41$ & 0.52 \\
\hline & $26(20.3 \%)$ & $20(16.4 \%)$ & $0.61^{a *}$ \\
\hline & 85 (66.4\%) & 85 (69.7\%) & $0.89^{*}$ \\
\hline & $2(1.6 \%)$ & $3(2.5 \%)$ & $0.96^{*}$ \\
\hline & 7 (5.5\%) & $5(4.1 \%)$ & $0.85^{*}$ \\
\hline & 0 & $3(2.5 \%)$ & $0.24^{*}$ \\
\hline & $8(6.3 \%)$ & $9(7.4 \%)$ & $0.94^{*}$ \\
\hline & $28.5 \pm 7.9$ & $28.8 \pm 9.6$ & 0.79 \\
\hline & $66.1 \pm 18.1$ & $63.8 \pm 17.6$ & 0.34 \\
\hline & $13.2 \pm 3.9$ & $13.0 \pm 4.0$ & 0.66 \\
\hline & $45.0 \pm 10.3$ & $47.1 \pm 10.2$ & 0.11 \\
\hline $\mathrm{AMl}$ & $59(46.1 \%)$ & 61 (50.0\%) & $0.80^{*}$ \\
\hline & 19 (14.8\%) & 20 (16.4\%) & $0.91^{*}$ \\
\hline & 41 (32.0\%) & 33 (27.1\%) & $0.61^{*}$ \\
\hline & 15 (11.7\%) & $13(10.7 \%)$ & $0.97^{*}$ \\
\hline & $22(17.2 \%)$ & $18(14.7 \%)$ & $0.78^{*}$ \\
\hline troke & $27(21.1 \%)$ & 29 (23.8\%) & $0.80^{*}$ \\
\hline 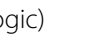 & $30 / 23$ & $17 / 22$ & $0.15^{*}$ \\
\hline & (23.4\%/17.9\%) & (13.9\%/18.0\%) & $(0.88)^{*}$ \\
\hline & $9(7.0 \%)$ & $8(6.5 \%)$ & $0.91^{*}$ \\
\hline
\end{tabular}

Atherosclerosis/ischemic HF/AMI

Congestive heart failure

Arterial hypertension

Obstructive pulmonary disease

Diabetes mellitus

Neurologic/psychiatric/post-stroke

Malignancy (solid/hematologic)

Pancreatitis 
Table 1 Baseline demographics/disease severity, and concomitant diseases (Continued)

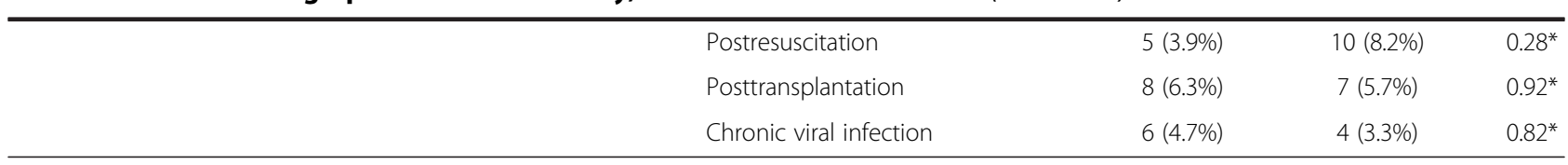

Data are given as means \pm SD, if not indicated otherwise. Independent samples $t$ test, except *a (chi-square), b (median, lQR).

other RRT modality in case of significant medical reasons. Switch of RRT modality was decided by the attending ICU physician in charge. In total, $19.5 \%$ patients randomized to receive IHD (group 1) versus $45.9 \%$ patients randomized to receive CVVH (group 2) were switched to the respective other modality during the study period. Time from both randomization and ICU admission until switch was not different between the two study groups: days from randomization until switch $4.4 \pm 12.0$ (group 1) versus $6.2 \pm 5.6$ (group 2), $P=0.37$. In the IHD group (group 1), switching of modality was necessary mainly due to reasons related to progressive hemodynamic instability (that is, advanced inotropic support) and/or due to significant fluid overload (16\%).

In group 2, switching of modality was mostly indicated because of repeated filter clotting (27\%), metabolic reasons (for example, progressive lactic acidosis; $18 \%)$, bleeding/discontinuation of anticoagulation required $(11 \%)$, severe thrombocytopenia $(5 \%)$, or because of desired mobilization/clinical improvement of study patients.

\section{Course of disease severity/organ dysfunction, key physiological and laboratory indices}

Disease severity was assessed over time by using SOFA and TISS-28 scoring systems, revealing no differences between the study groups (Table 2). Cumulative catecholamine need and total days on vasopressors were not different between the two study groups (data not shown). Key hemodynamic variables (that is, heart rate, mean arterial pressure, and central venous pressure) did not differ between the study groups at randomization, baseline, and during follow-up (Tables 1 and 2). Need for mechanical ventilation was assessed in both study groups. Initial need for mechanical ventilation (Table 1), total days on mechanical ventilation (Table 3), and $\mathrm{paO}_{2} / \mathrm{FiO}_{2}$ (data not shown) did not differ between the study groups. During follow-up, other physiological variables including blood $\mathrm{pH}$, urinary output, temperature, and $\mathrm{C}$-reactive protein were also not different (Table 2).

Survival at 14 days after RRT, 14-day-, 30-day- and all-cause mortality data

Survival at 14 days after RRT, 14-day-, 30-day- and allcause intrahospital mortality rates were not different between the study groups in both the ITT and PP populations (all n.s.; survival rates of the ITT population given in
Table 3). The univariate OR (for patients in IHD group) for survival at 14 days after end of RRT was 0.84 (95\% CI, 0.49 to $1.41 ; P=0.5$ ). OR (for patients in IHD group) for 14-day mortality was 1.27 (95\% CI, 0.76 to $-2.12 ; P=0.36$ ). Missing data (survival at 14 days after RRT) in nine (IHD group) versus eight (CVVH group) patients (that is, $7.0 \%$, n.s.). OR (IHD group) for 28-day mortality was 1.37 (95\% CI, 0.82 to $2.27 ; P=0.22$ ). For patients randomized to receive IHD, the OR for 30-day mortality was 1.32 (95\% CI, 0.80 to $2.19 ; P=0.27)$, and for all-cause intrahospital mortality, 1.26 (95\% CI, 0.76 to $2.10 ; P=0.37$ ). In the CVVH study group, the survival rate at 14 days after RRT was $4.4 \%$ higher, and mortality rates were not significantly reduced by $5.8 \%$ (14-day mortality), 7.9\% (28-day mortality), $7.0 \%$ (30-day mortality), and 5.7\% (all-cause intrahospital mortality).

To investigate the potential impact of modality switch on outcome indices, subanalyses were performed comparing patients who switched with those who stayed on the initial therapy, revealing no influence on the primary outcome measure, 14-day mortality, 30-day mortality, or all-cause intrahospital mortality (all $P=$ n.s., data not shown).

Moreover, we investigated whether higher norepinephrine need (defined as $>0.3 \mu \mathrm{g} / \mathrm{kg} / \mathrm{min}$ at any time during the study interval) was associated with different outcomes in the respective study groups. It was noted that, in this subgroup with higher catecholamine need ( $n=47$ in group 1 versus $n=40$ in group 2), survival at 14 days after RRT (36.2\% versus $37.5 \%$; $P=0.9$ ), 14-day-, 30-day-, and all-cause mortality rates $(65.9 \%$ versus $60.0 \% ; P=$ 0.92 ) were also not different between the study groups. To illustrate survival characteristics, Kaplan-Meier survival estimates were constructed for the total study population (Figure 2) and the subpopulation receiving a higher vasopressor dose (Figure 3).

\section{Discussion}

Acute renal failure (ARF) remains an important determinant for patient outcomes and constitutes a particular burden for healthcare systems worldwide [1]. Here we investigate the potential impact of two of the major RRT modalities (that is, IHD and CVVH) on mortality and renal-related outcome measures in a single-center prospective randomized controlled trial (CONVINT). After inclusion of 252 patients with ARF requiring RRT, we observed no statistically significant differences in 14-day, 
Table 2 Course of disease severity, physiological, and laboratory indices

\begin{tabular}{|c|c|c|c|c|c|c|c|c|c|c|}
\hline & Range, units & Group & Day 1 & Day 3 & Day 5 & Day 7 & Day 10 & Day 15 & Day 21 & $\begin{array}{c}P \text { value } \\
\text { Day } 1 \text { (day 10) }\end{array}$ \\
\hline \multirow[t]{4}{*}{ SOFA score } & - & $\mathrm{IHD}$ & 13.3 & 13.0 & 11.5 & 10.1 & 7.6 & 8.3 & 5.8 & n.s. (n.s.) \\
\hline & & & \pm 3.7 & \pm 5.1 & \pm 4.6 & \pm 5.2 & \pm 5.0 & \pm 4.3 & \pm 0.8 & \\
\hline & & $\mathrm{CWH}$ & 13.0 & 12.2 & 10.6 & 9.5 & 8.2 & 8.4 & 4.7 & \\
\hline & & & \pm 4.0 & \pm 3.9 & \pm 4.2 & \pm 5.2 & \pm 5.1 & \pm 4.4 & \pm 7.2 & \\
\hline \multirow[t]{4}{*}{ TISS-28 score } & - & $\mathrm{IHD}$ & 45.3 & 42.0 & 41.2 & 37.8 & 29.9 & 35.8 & 37.6 & n.s. (n.s.) \\
\hline & & & \pm 9.5 & \pm 9.1 & \pm 9.7 & \pm 8.0 & \pm 9.0 & \pm 6.1 & \pm 5.5 & \\
\hline & & $\mathrm{CWH}$ & 46.8 & 43.3 & 39.9 & 37.3 & 33.8 & 40.4 & 25.3 & \\
\hline & & & \pm 10.2 & \pm 8.3 & \pm 9.1 & \pm 12.3 & \pm 10.8 & \pm 7.9 & \pm 17.2 & \\
\hline \multirow[t]{4}{*}{ Serum creatinine } & $<1.20 \mathrm{mg} / \mathrm{dl}$ & $\mathrm{IHD}$ & 3.68 & 3.29 & 3.17 & 2.82 & 2.32 & 2.54 & 2.70 & n.s. (n.s.) \\
\hline & & & \pm 1.86 & \pm 2.59 & \pm 1.61 & \pm 1.51 & \pm 1.40 & \pm 1.40 & \pm 1.54 & \\
\hline & & $\mathrm{CWH}$ & 3.74 & 2.18 & 2.31 & 2.08 & 1.93 & 1.85 & 1.86 & \\
\hline & & & \pm 1.88 & \pm 1.19 & \pm 1.36 & \pm 1.40 & \pm 1.34 & \pm 1.42 & \pm 1.52 & \\
\hline \multirow[t]{4}{*}{ Serum urea } & $14-46$ mg/dl & $\mathrm{IHD}$ & 164.8 & 121.5 & 125.4 & 124.9 & 105.9 & 120.4 & 117.6 & n.s. (n.s.) \\
\hline & & & \pm 83.4 & \pm 55.8 & \pm 50.0 & \pm 48.9 & \pm 53.9 & \pm 48.9 & \pm 60.4 & \\
\hline & & $\mathrm{CWH}$ & 155.7 & 93.4 & 105.1 & 107.5 & 94.4 & 100.9 & 94.4 & \\
\hline & & & \pm 70.0 & \pm 44.3 & \pm 53.2 & \pm 48.9 & \pm 48.3 & \pm 53.3 & \pm 65.7 & \\
\hline \multirow[t]{4}{*}{ Urinary output (24 hours) } & $>500 \mathrm{ml}$ & $\mathrm{IHD}$ & 922 & 944 & 914 & 1,331 & 1,714 & 1,376 & 3,570 & n.s. (n.s.) \\
\hline & & & $\pm 1,299$ & $\pm 1,464$ & $\pm 1,415$ & $\pm 1,624$ & $\pm 1,338$ & $\pm 1,508$ & $\pm 3,614$ & \\
\hline & & $\mathrm{CWH}$ & 649 & 823 & 1,402 & 1,645 & 1,567 & 1,591 & 1,964 & \\
\hline & & & \pm 948 & $\pm 1,323$ & $\pm 1,785$ & $\pm 1,636$ & $\pm 1,474$ & $\pm 2,093$ & \pm 757 & \\
\hline \multirow[t]{4}{*}{$\mathrm{pH}$} & $7.35-7.45$ & $\mathrm{IHD}$ & 7.35 & 7.37 & 7.38 & 7.37 & 7.39 & 7.36 & 7.39 & n.s. (n.s.) \\
\hline & & & \pm 0.1 & \pm 0.1 & \pm 0.1 & \pm 0.1 & \pm 0.1 & \pm 0.1 & \pm 0.1 & \\
\hline & & $\mathrm{CWH}$ & 7.36 & 7.37 & 7.40 & 7.38 & 7.37 & 7.39 & 7.39 & \\
\hline & & & \pm 0.1 & \pm 0.1 & \pm 0.1 & \pm 0.2 & \pm 0.1 & \pm 0.1 & \pm 0.2 & \\
\hline \multirow[t]{4}{*}{ Heart rate } & 80-100 bpm & $\mathrm{IHD}$ & 104.0 & 93.8 & 90.9 & 92.9 & 86.9 & 85.5 & 86.5 & n.s. (n.s.) \\
\hline & & & \pm 26.1 & \pm 23.3 & \pm 20.3 & \pm 17.0 & \pm 16.4 & \pm 19.9 & \pm 12.9 & \\
\hline & & $\mathrm{CWH}$ & 105.2 & 93.6 & 90.0 & 87.5 & 89.7 & 93.1 & 96.5 & \\
\hline & & & \pm 21.3 & \pm 22.3 & \pm 20.4 & \pm 19.5 & \pm 18.1 & \pm 17.2 & \pm 15.4 & \\
\hline \multirow[t]{4}{*}{ Mean arterial pressure } & $50-80 \mathrm{~mm} \mathrm{Hg}$ & $\mathrm{IHD}$ & 73.3 & 76.5 & 76.9 & 82.2 & 78.1 & 83.3 & 70.0 & n.s. (n.s.) \\
\hline & & & \pm 16.5 & \pm 17.6 & \pm 15.9 & \pm 16.7 & \pm 17.2 & \pm 11.9 & \pm 15.1 & \\
\hline & & $\mathrm{CWH}$ & 72.0 & 76.2 & 76.6 & 77.7 & 81.2 & 75.5 & 75.3 & \\
\hline & & & \pm 14.1 & \pm 13.8 & \pm 12.7 & \pm 12.4 & \pm 14.9 & \pm 25.9 & \pm 16.5 & \\
\hline \multirow[t]{4}{*}{ Central venous pressure } & 8-12 mm Hg & $\mathrm{HD}$ & 14.7 & 12.9 & 12.2 & 12.2 & 11.1 & 10.7 & 12.9 & n.s. (n.s.) \\
\hline & & & \pm 5.3 & \pm 4.9 & \pm 3.9 & \pm 4.8 & \pm 3.9 & \pm 3.4 & \pm 2.94 & \\
\hline & & $\mathrm{CWH}$ & 14.3 & 13.6 & 11.8 & 10.9 & 12.3 & 11.5 & 11.9 & \\
\hline & & & \pm 5.4 & \pm 5.6 & \pm 4.2 & \pm 3.8 & \pm 4.6 & \pm 3.6 & 3.0 & \\
\hline \multirow[t]{4}{*}{ Temperature } & $36.5^{\circ} \mathrm{C}-37.5^{\circ} \mathrm{C}$ & $\mathrm{IHD}$ & 37.5 & 37.4 & 37.2 & 37.4 & 37.2 & 37.7 & 37.7 & n.s. (n.s.) \\
\hline & & & \pm 1.48 & \pm 1.03 & \pm 1.3 & \pm 0.65 & \pm 1.6 & \pm 0.67 & \pm 0.7 & \\
\hline & & $\mathrm{CWH}$ & 37.5 & 37.1 & 37.1 & 37.1 & 37.2 & 37.1 & 37.7 & \\
\hline & & & \pm 1.30 & \pm 1.16 & \pm 0.94 & \pm 0.90 & \pm 0.91 & \pm 1.05 & \pm 1.02 & \\
\hline \multirow[t]{4}{*}{ C-reactive protein } & $<0.50 \mathrm{mg} / \mathrm{dl}$ & $\mathrm{IHD}$ & 18.0 & 15.6 & 10.5 & 10.5 & 8.3 & 7.14 & 11.7 & n.s. (n.s.) \\
\hline & & & \pm 10.0 & \pm 10.1 & \pm 8.3 & \pm 7.7 & \pm 6.7 & \pm 4.95 & \pm 8.25 & \\
\hline & & $\mathrm{CWH}$ & 18.8 & 15.9 & 10.1 & 9.4 & 10.0 & 12.6 & 10.8 & \\
\hline & & & \pm 10.3 & \pm 9.5 & \pm 8.55 & \pm 7.2 & \pm 7.1 & \pm 7.5 & \pm 7.6 & \\
\hline
\end{tabular}


Table 3 Clinical outcome of study patients

\begin{tabular}{|c|c|c|c|c|}
\hline & & iHD group & CVVH group & $P$ value \\
\hline & & $(n=128)$ & $(n=122)$ & \\
\hline Survival at 14 days after RRT & & $39.5 \%$ & $43.9 \%$ & $0.81^{\mathrm{a}}$ \\
\hline 14-day mortality rate & & $43.6 \%$ & $37.8 \%$ & $0.63^{\mathrm{a}}$ \\
\hline 30-day mortality rate & & $52.4 \%$ & $45.4 \%$ & $0.60^{\mathrm{a}}$ \\
\hline All-cause intrahospital mortality rate (last contact) & & $60.3 \%$ & $54.6 \%$ & $0.72^{\mathrm{a}}$ \\
\hline Days until death & & $15.6 \pm 44.5$ & $18.5 \pm 48.9$ & 0.71 \\
\hline Days until death on ICU & & $15.5 \pm 45.9$ & $18.4 \pm 50.0$ & 0.73 \\
\hline Days until hospital discharge (in survivors) & & $51.2 \pm 47.1$ & $48.7 \pm 49.7$ & 0.78 \\
\hline Days in ICU & & $25.2 \pm 40.1$ & $22.3 \pm 26.1$ & 0.50 \\
\hline Days in hospital & & $33.9 \pm 49.3$ & $32.4 \pm 37.4$ & 0.79 \\
\hline \multirow[t]{6}{*}{ Suspected reason for death (multiple possible) } & Cardiac failure & 31 & 22 & $0.42^{\mathrm{a}}$ \\
\hline & Pulmonary failure & 39 & 31 & $0.59^{\mathrm{a}}$ \\
\hline & Sepsis & 56 & 45 & $0.55^{\mathrm{a}}$ \\
\hline & CNS & 7 & 6 & $0.92^{\mathrm{a}}$ \\
\hline & Hemorrhagy & 5 & 4 & $0.93^{\mathrm{a}}$ \\
\hline & Withdrawal of therapy & 4 & 2 & $0.74^{\mathrm{a}}$ \\
\hline Days on RRT & & $17.2 \pm 37.1$ & $13.7 \pm 17.9$ & 0.35 \\
\hline Dialysis-free days & & $4.2 \pm 9.6$ & $3.1 \pm 9.0$ & 0.38 \\
\hline RRT switch (number of patients) & & $25(19.5 \%)$ & $56(45.9 \%)$ & $0.002^{\mathrm{a}}$ \\
\hline \multirow{2}{*}{$\begin{array}{l}\text { Number of patients on RRT (\% of survivors, days } \\
\text { after ICU admission) }\end{array}$} & At 21 days & $20(32.3 \%)$ & $20(29.9 \%)$ & $0.97^{\mathrm{a}}$ \\
\hline & At 60 days & $14(26.4 \%)$ & $13(22.8 \%)$ & $0.90^{\mathrm{a}}$ \\
\hline $\begin{array}{l}\text { Serum creatinine at hospital discharge/last contact } \\
\text { (in survivors; } \mathrm{mg} / \mathrm{dl} \text { ) }\end{array}$ & & $2.18 \pm 1.8$ & $2.12 \pm 1.7$ & 0.85 \\
\hline Total days on vasopressors & & $4.3 \pm 3.7$ & $4.5 \pm 3.7$ & 0.75 \\
\hline \multirow[t]{3}{*}{ Cumulative vasopressor dose (g) } & Epinephrine & 0.70 & 0.64 & 0.96 \\
\hline & Norepinephrine & 19.1 & 18.5 & 0.30 \\
\hline & Dobutamine & 150.2 & 137.9 & 0.56 \\
\hline Total days on mechanical ventilation & & $8.1 \pm 8.8$ & $7.2 \pm 6.5$ & 0.34 \\
\hline Total fluid balance $(\mathrm{L})$ & & $20.5 \pm 23.2$ & $24.9 \pm 28.4$ & 0.19 \\
\hline
\end{tabular}

Between-group $P$ values are given. Independent samples $t$ test except ${ }^{a}\left(x^{2}\right)$. Means \pm SD.

30-day, and all-cause mortality, renal-related outcome measures, or survival at 14 days after RRT. Recent metaanalyses call for larger RCTs because of limited sample sizes of previous clinical trials and considerable heterogeneity of respective study populations [3,11-15]. Our data support findings from previous studies that intermittent and continuous RRT (CRRT) modalities may be considered equivalent approaches in a general population of critically ill patients with dialysis-dependent ARF.

Our study population consisted of a cohort of critically ill medical ICU patients with persisting ARF requiring RRT, despite adequate hemodynamic status. At study day 1, the mean APACHE II score in the overall sample was $30.4 \pm 7.1$. The majority of patients initially presented with established multiple organ failure. The major underlying condition for development of multiple organ failure at baseline was severe sepsis/septic shock. Besides requiring renal support, most patients required mechanical ventilation and vasopressor support also (please refer to Table 1). This may explain the rather high mortality rates observed in our trial (Table 3, Figure 2). In line with previously published larger clinical trials in patients with ARF, severe sepsis/septic shock due to pneumogenic or abdominal sepsis was the major underlying pathology in our study population.

In CONVINT, we deliberately chose to exclude patients with preexisting advanced chronic renal failure (that is, patients with previous serum creatinine values of $>3 \mathrm{mg} / \mathrm{dl}$ ). Patients in CONVINT received daily IHD for at least 4 hours. In CVVH-treated patients prescribed "dose" (that is, filtration rate was $35 \mathrm{ml} / \mathrm{kg} / \mathrm{h}$ based on the landmark trial by Ronco et al. [22] with a 


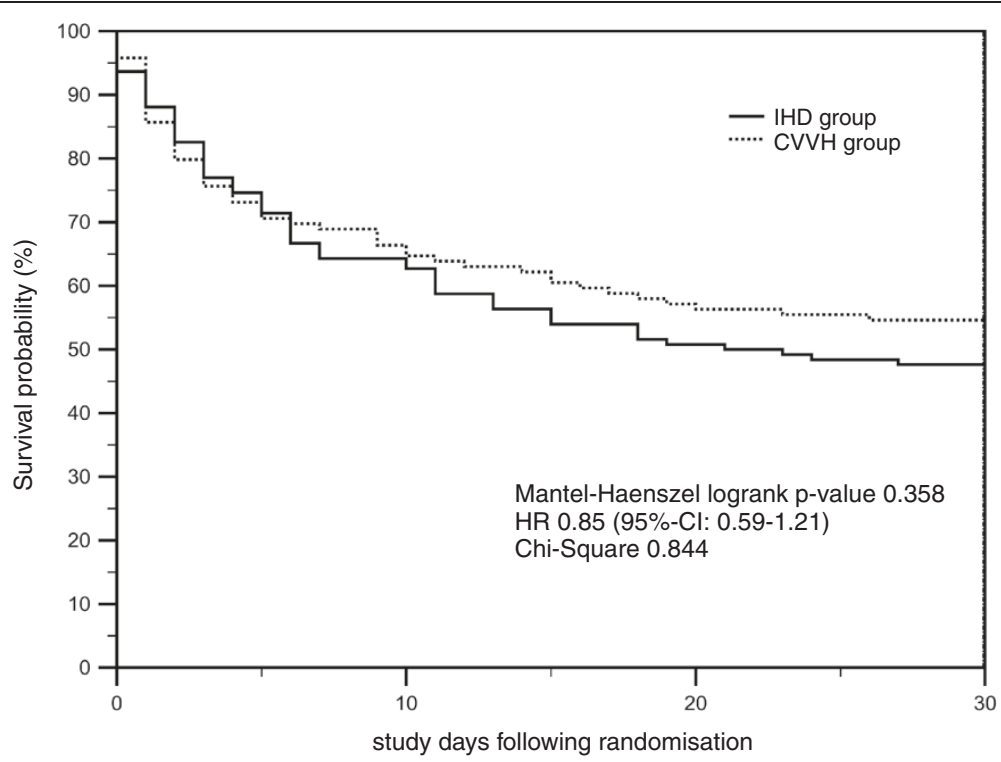

Figure 2 Kaplan-Meier survival estimates for patients randomized to IHD (full line, $n=128$ ) and CVVH (dotted line, $n=122$ ) are illustrated (total study population). Mantel-Haenszel log-rank $P$ value, hazard ratio (HR) including $95 \% \mathrm{Cl}$ and $x^{2}$ is given.

delivered dose of $30.9 \pm 7.0 \mathrm{ml} / \mathrm{kg} /$ hour). This discrepancy is in line with previous publications [23] demonstrating that the applied dose may differ from the prescribed dose in critically ill patients. This must be kept in mind when prescribing CRRT.

Until recently, however, the optimal dose of CRRT in critically ill patients with ARF was controversial. Data from two recent large-scale multicenter RCTs now demonstrate that filtration rates above $25 \mathrm{ml} / \mathrm{kg} /$ hour may not further improve the outcome of critically ill patients with ARF $[24,25]$. Thus, the filtration rate actually applied in CONVINT could be considered adequate on the basis of recent recommendations [26].

As recent guidelines [26] also specifically suggest preferential use of CRRT in hemodynamically unstable patients, we also investigated the effect of IHD versus CVVH in the subgroup of patients with relevant vasopressor requirements. In the overall study population,

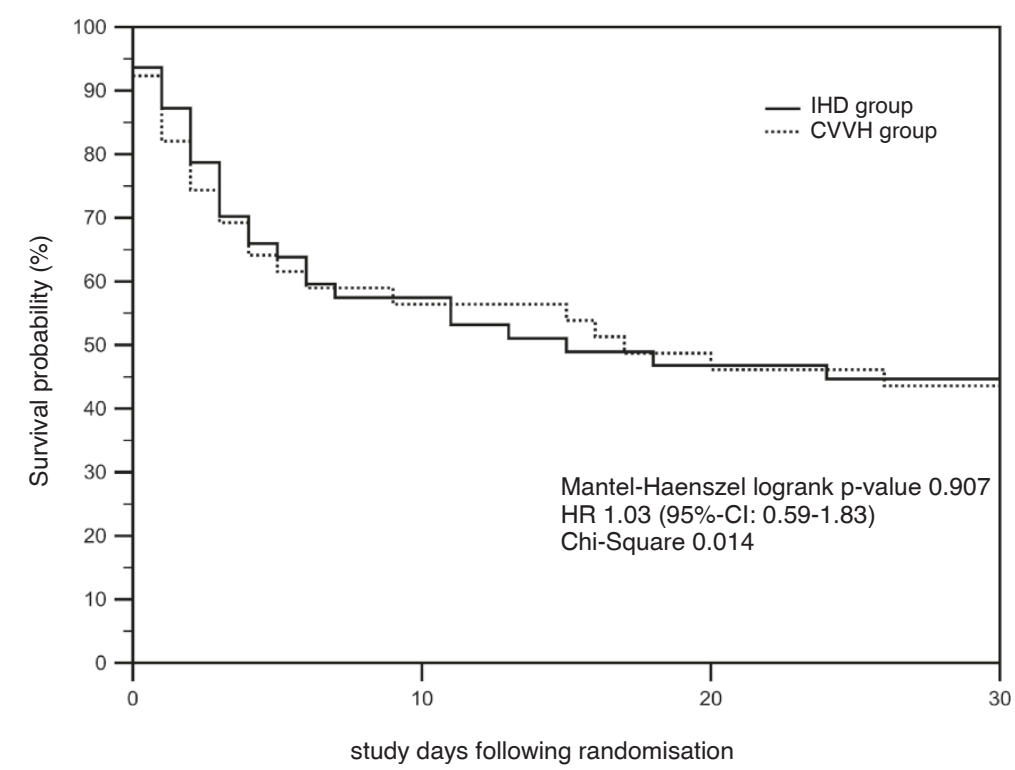

Figure 3 Kaplan-Meier survival estimates for the subpopulation of patients with high vasopressor need (overall sample; high vasopressor use defined as $>0.3 \mu \mathrm{g} / \mathrm{kg} / \mathrm{min}$ at any point in time during the study interval) in the IHD (full line, $n=47$ ) versus CVVH (dotted line, $\boldsymbol{n}=\mathbf{4 0}$ ) groups are illustrated. Mantel-Haenszel log-rank $P$ value, hazard ratio (HR), including $95 \% \mathrm{Cl}$ and $x^{2}$ is given. 
mortality rates or survival at 14 days after RRT in patients with norepinephrine need of $>0.3 \mu \mathrm{g} / \mathrm{kg} / \mathrm{min}$ were not different between the two study groups (Figure 3), however, the number of study patients in this subgroup was rather small. This finding should therefore not be used as an argument against the preferential use of CRRT in hemodynamically compromised individuals. As recommended by the KDIGO guideline [26], priority should be given to individualized therapeutic decisions that are made by ICU specialists or consulting nephrologists on the basis of the specific clinical and hemodynamic status of the patient.

A number of limitations of our analysis require discussion. First, the initial power calculation aimed at inclusion of 200 patients per arm. CONVINT was terminated early after inclusion of 252 patients (63\% of the targeted population). The reason for early trial termination was a major change in RRT equipment and procedures that was beyond the investigators' control. With the ICU team confronted with new machinery and new treatment protocols (for instance, automated citrate anticoagulation, preference for HDF instead of HD), it became obvious that this would introduce an unacceptable bias to the trial. The investigators therefore decided to terminate the study prematurely.

After termination of the trial, a retrospective explorative analysis was performed to determine whether the trial might have been able to reach statistical significance regarding the primary end point if continued as planned. This was, however, not the case, as the between-group $P$ value for the primary outcome measure would not have reached statistical significance in the unlikely event that survival rates at 14 days after RRT in the 148 remaining cases had differed between groups by an additional $30 \%$. However, by definition, we cannot rule out an effect of underpowering and related beta error on the outcome measures (including renal outcome measures) after early trial termination.

Second, switching of RRT modality in cases of significant RRT modality-related complications or medical reasons was deliberately allowed in the study protocol. This was done for safety reasons and was considered inevitable in this cohort of critically ill patients. Although the substantial number of cross-overs could have influenced our overall findings, differences in both the primary end point and respective mortality rates in the subgroups of patients switched/not switched were not observed.

Third, we present data from a single-center RCT, and the overall trial period was rather long. Thus, the inherent limitations of monocentric trials should be kept in mind when analyzing the data provided. Nevertheless, as therapeutic standards of care were unchanged during the trial period, we believe that the total study interval should be a minor limitation of our analysis.
Fourth, we defined a previous serum creatinine value of $>3 \mathrm{mg} / \mathrm{dl}$ as an exclusion criterion to exclude patients with advanced chronic renal failure. Conversely, patients with moderate to advanced chronic renal insufficiency could be included in the study and might have influenced our data.

\section{Conclusions}

In conclusion, after recruitment of 252 patients with dialysis-dependent ARF to a prospective single-center randomized controlled trial, we observed no statistically significant differences regarding 14-day-, 30-day-, allcause intrahospital mortality, renal-related outcome measures, or survival at 14 days after RRT. Moreover, no significant effect of the RRT modality on the percentage of surviving patients still requiring RRT at days 21 or 60 was observed. Our data lend further support to the view that intermittent and continuous RRT may be considered equivalent approaches for a general population of critically ill patients with dialysis-dependent ARF treated in a medical ICU.

\section{Key messages}

- In critically ill patients with dialysis-dependent ARF, statistically significant differences regarding mortality rates, renal-related outcome measures, or the rate of surviving patients at 14 days after end of RRT were not observed between patients randomized to receive either daily iHD or $\mathrm{CVVH}$.

- In the subgroup of patients with higher catecholamine need, survival at 14 days after RRT, 14-day, 30-day, and all-cause mortality rates were also not different between the two RRT modalities (that is, daily iHD versus CVVH).

- Our data support the view that intermittent and continuous RRT may be considered equivalent approaches for a general population of critically ill patients with dialysis-dependent ARF treated in a medical ICU.

\section{Abbreviations}

ARF: Acute renal failure; $\mathrm{CWH}$ : continuous venovenous hemofiltration; iHD: intermittent hemodialysis; KDIGO: Kidney Disease: Improving Globa Outcomes; RRT: renal replacement therapy.

Competing interests

The authors declare that they have no conflict of interest, financially or otherwise, to disclose.

Authors' contributions

JCS, SVH, RP, TOB, DH, and AJ designed, supervised, and analyzed all data. JCS, RP, and AJ wrote the manuscript. CB and SB acquired data and analyzed all data. All authors read and approved the final version of the manuscript.

\section{Acknowledgements}

The CONVINT investigators are indebted to all ICU nurses, staff, and doctors as well as to all laboratory personnel for their continued efforts and 
outstanding support. The CONVINT investigators also thank Prof. Dr. Dipl. mat. J. Högel, Department of Biometry and Medical Documentation, University of UIm, Ulm, Germany (present address: Department of Human Genetics, University of Ulm, Ulm, Germany), for provision of statistical support/establishment of the randomization procedure. Furthermore, the authors are indebted to Evelyn Kuhnt, expert statistician of the Zentrum für Klinische Studien, Leipzig, Germany, for analyzing the power/precision of the CONVINT data in the context of early trial termination.

\section{Author details}

'Department of Nephrology and Medical Intensive Care, Charité- Universitätsmedizin Berlin, Campus Virchow-Klinikum, Berlin, Germany. ${ }^{2}$ Department of Clinical Cardiology, Charité-

Universitätsmedizin Berlin, Campus Virchow-Klinikum, Berlin, Germany. ${ }^{3}$ Department of Gastroenterology, Charité- Universitätsmedizin Berlin, Campus Virchow-Klinikum, Berlin, Germany.

Received: 12 May 2013 Accepted: 3 January 2014

Published: 10 January 2014

\section{References}

1. Bellomo R, Kellum JA, Ronco C: Acute kidney injury. Lancet 2012, 380:756-766

2. Druml W: Acute renal failure is not a "cute" renal failure! Intensive Care Med 2004, 30:1886-1890

3. Pannu N, Klarenbach S, Wiebe N, Manns B, Tonelli M: Renal replacement therapy in patients with acute renal failure: a systematic review. JAMA 2008, 299:793-805

4. Ricci Z, Ronco C: Timing, dose and mode of dialysis in acute kidney injury. Curr Opin Crit Care 2011, 17:556-561.

5. Augustine JJ, Sandy D, Seifert TH, Paganini EP: A randomized controlled trial comparing intermittent with continuous dialysis in patients with ARF. Am J Kidney Dis 2004, 44:1000-1007.

6. John S, Griesbach D, Baumgartel M, Weihprecht H, Schmieder RE, Geiger H: Effects of continuous haemofiltration vs intermittent haemodialysis on systemic haemodynamics and splanchnic regional perfusion in septic shock patients: a prospective, randomized clinical trial. Nephrol Dial Transplant 2001, 16:320-327.

7. Lins RL, Elseviers MM, Van der Niepen P, Hoste E, Malbrain ML, Damas P, Devriendt J: Intermittent versus continuous renal replacement therapy for acute kidney injury patients admitted to the intensive care unit: results of a randomized clinical trial. Nephrol Dial Transplant 2009, 24:512-518.

8. Mehta RL, McDonald B, Gabbai FB, Pahl M, Pascual MT, Farkas A, Kaplan RM: A randomized clinical trial of continuous versus intermittent dialysis for acute renal failure. Kidney Int 2001, 60:1154-1163.

9. Uehlinger DE, Jakob SM, Ferrari P, Eichelberger M, Huynh-Do U, Marti HP, Mohaupt MG, Vogt B, Rothen HU, Regli B, et al: Comparison of continuous and intermittent renal replacement therapy for acute renal failure. Nephrol Dial Transplant 2005, 20:1630-1637.

10. Vinsonneau C, Camus C, Combes A, de Beauregard MAC, Klouche K, Boulain $T$, Pallot $J L$, Chiche JD, Taupin P, Landais P, et al: Continuous venovenous haemodiafiltration versus intermittent haemodialysis for acute renal failure in patients with multiple-organ dysfunction syndrome: a multicentre randomised trial. Lancet 2006, 368:379-385.

11. Bagshaw SM, Berthiaume LR, Delaney A, Bellomo R: Continuous versus intermittent renal replacement therapy for critically ill patients with acute kidney injury: a meta-analysis. Crit Care Med 2008, 36:610-617.

12. Bansal VB: Comparison of intermittent hemodialysis (IHD) and continuous renal replacement therapy (CRRT) in acute renal failure (ARF): meta-analysis of published data. J Am Soc Nephrol 1999, 10:137A.

13. Friedrich JO, Wald R, Bagshaw SM, Burns KE, Adhikari NKJ: Hemofiltration compared to hemodialysis for acute kidney injury: systematic review and meta-analysis. Crit Care 2012, 16:R146.

14. Ghahramani N, Shadrou S, Hollenbeak C: A systematic review of continuous renal replacement therapy and intermittent haemodialysis in management of patients with acute renal failure. Nephrology (Carlton) 2008, 13:570-578.

15. Rabindranath $\mathrm{K}$, Adams J, Macleod AM, Muirhead N: Intermittent versus continuous renal replacement therapy for acute renal failure in adults. Cochrane Database Syst Rev 2007, 3:CD003773.

16. Honore PM, Jacobs R, Joannes-Boyau O, De Regt J, Boer W, De Waele E, Collin V, Spapen HD: Septic AKI in ICU patients. diagnosis, pathophysiology, and treatment type, dosing, and timing: a comprehensive review of recent and future developments. Ann Intensive Care 2011, 1:32.

17. Schwenger V, Weigand MA, Hoffmann O, Dikow R, Kihm LP, Seckinger J, Miftari N, Schaier M, Hofer S, Haar C, et al: Sustained low efficiency dialysis using a single-pass batch system in acute kidney injury: a randomized interventional trial: the REnal Replacement Therapy Study in Intensive Care Unit Patients. Crit Care 2012, 16:R140.

18. Vincent $\mathrm{J}$, Moreno R, Takala J, Willatts S, De Mendonca A, Bruining H, Reinhart CK, Suter PM, Thijs LG: The SOFA (Sepsis-related Organ Failure Assessment) score to describe organ dysfunction/failure. On behalf of the Working Group on Sepsis-Related Problems of the European Society of Intensive Care Medicine. Intensive Care Med 1996, 22:707-710.

19. Miranda DR, de Rijk A, Schaufeli W: Simplified Therapeutic Intervention Scoring System: the TISS-28 items: results from a multicenter study. Crit Care Med 1996, 24:64-73.

20. Knaus WA, Draper EA, Wagner DP, Zimmerman JE: APACHE II: a severity of disease classification system. Crit Care Med 1985, 13:818-829.

21. Le Gall JR, Lemeshow S, Saulnier F: A new Simplified Acute Physiology Score (SAPS II) based on a European/North American multicenter study. JAMA 1993, 270:2957-2963.

22. Ronco C, Bellomo R, Homel P, Brendolan A, Dan M, Piccinni P, La Greca G: Effects of different doses in continuous veno-venous haemofiltration on outcomes of acute renal failure: a prospective randomised trial. Lancet 2000, 356:26-30

23. Vesconi S, Cruz DN, Fumagalli R, Kindgen-Milles D, Monti G, Marinho A, Mariano F, Formica M, Marchesi M, Rene R, et al: Delivered dose of renal replacement therapy and mortality in critically ill patients with acute kidney injury. Crit Care 2009, 13:R57.

24. Bellomo R, Cass A, Cole L, Finfer S, Gallagher M, Lo S, McArthur C, McGuinness S, Myburgh J, Norton R, et al: Intensity of continuous renal-replacement therapy in critically ill patients. N Engl J Med 2009, 361:1627-1638

25. Palevsky PM, Zhang JH, O'Connor TZ, Chertow GM, Crowley ST, Choudhury D, Finkel K, Kellum JA, Paganini E, Schein RM, et al: Intensity of renal support in critically ill patients with acute kidney injury. N Engl J Med 2008, 359:7-20.

26. Disease K, Improving Global Outcomes (KDIGO) Acute Kidney Injury Work Group: KDIGO Clinical Practice Guideline for Acute Kidney Injury. Kidney Int 2012, 2:1-138.

doi:10.1186/cc13188

Cite this article as: Schefold et al:: The effect of continuous versus intermittent renal replacement therapy on the outcome of critically ill patients with acute renal failure (CONVINT): a prospective randomized controlled trial. Critical Care 2014 18:R11.

\section{Submit your next manuscript to BioMed Central and take full advantage of:}

- Convenient online submission

- Thorough peer review

- No space constraints or color figure charges

- Immediate publication on acceptance

- Inclusion in PubMed, CAS, Scopus and Google Scholar

- Research which is freely available for redistribution 